\title{
Dynamics of Fluctuations for Quantum Lattice Systems
}

\author{
D. Goderis $\star$ A. Verbeure and P. Vets $\star \star$ \\ Instituut voor Theoretische Fysica, Universiteit Leuven, B-3030 Leuven, Belgium
}

\begin{abstract}
For short range interactions and for $L^{1}$-space clustering states it is proved that there exists a bonafide time evolution on the set of normal fluctuations. This dynamics is applied to derive the notion of equilibrium state of the algebra of fluctuations.
\end{abstract}

\section{Introduction}

The aim of statistical mechanics is the explanation of the macrophenomena on the basis of the microstructure. For this it is important to make a clear distinction between microscopic observables, states, etc. and macroscopic ones.

In the algebraic approach to quantum statistical mechanics the microscopic system is currently described by a ( $C^{*}$ - or von Neumann-) algebra $\mathscr{B}$ of observables. A physical state is a positive linear normalized functional $\omega$ of $\mathscr{B}$ and a dynamics of the system $(\mathscr{B}, \omega)$ is a one-parameter group $\alpha_{t}$ of $*$-automorphisms of $\mathscr{B}$.

Analogously any macroscopic system should be described by an analogous triplet of observables, states and dynamics.

The problem is to construct by precise procedures the macroscopic triplets out of the microscopic ones.

A well known [1] algebra of macroscopic observables is the one given by the observables at infinity and consisting of the space means of local micro-observables: i.e. for $A$ any local observable, one considers the corresponding observable at infinity,

$$
A_{\omega}^{\infty}=\lim _{V \rightarrow \infty} \frac{1}{V} \int_{V} d x \tau_{x} A,
$$

where $V$ is any finite volume and $\tau_{x}$ the translation over the space variable $x$. In probability this limit is known as the law of large numbers. The algebra generated by the limits $\left\{A_{\omega}^{\infty} \mid A \in \mathscr{B}\right\}$ is an abelian algebra of macroscopic observables.

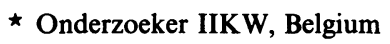

$\star \star$ Onderzoeker IIKW, Belgium 
Here we are concerned with another algebra of macro-observables, namely this which is generated by the limit points under the central limit, i.e. one considers:

$$
B_{\omega}(A)=\lim _{V \rightarrow \infty} \frac{1}{\sqrt{V}} \int_{V} d x\left(\tau_{x} A-\omega(A)\right) .
$$

In physics, these limits are called (normal) fluctuations. For quantum systems the existence of these limits is studied in $[2,3]$ for mean fields, respectively mixing systems. In Sect. 3 we give a new version of the main theorem of [3] about the existence of normal fluctuations under a slightly more stringent, but more transparent cluster condition. We prove that the set of central limits from the microscopic algebra generates a representation of a CCR-algebra induced by a quasi-free state. Under the central limit the algebra $\mathscr{B}$ of micro-observables is mapped into a CCR-algebra $\tilde{\mathscr{B}}$ of macroscopic fluctuations (for the precise formulations see below). This map is clearly not injective. This is the phenomenon of coarse graining. A mathematically rigorous description of this property is given in Theorem 3.4. Also under the central limit, the microstate $\omega$ is mapped into a quasi-free state $\tilde{\omega}$.

In this paper we concentrate on the study of the dynamics of the fluctuations $\widetilde{B}$ for interacting systems. For some mean field models this time evolution has been computed before $[4,5]$ and for general mean field systems $[2,6]$. Here we perform this program for quantum lattice systems with a quasi-local time evolution, induced by short range interactions and for states satisfying a $L^{1}$-cluster condition for space translations.

Free particles and mean field systems have the particular property that strictly local observables are mapped into strictly local observables. This means that if the fluctuations exist for local observables, then they also exist for their time evolved ones. This is not true anymore for interacting systems, the time evolution is destroying the strict locality. The main technical problem is to control this time evolution and to show that one can pass it through the central limit, in order to get a basis for a consistent dynamics of fluctuations. This is proved in Sect. 4 and we end up with the existence and explicit construction of a nontrivial, reversible dynamics $\tilde{\alpha}_{t}$ on the CCR-algebra $\tilde{\mathscr{B}}$ of fluctuations. This dynamics is fully determined by the microscopic one and is of the so-called quasi-free type, i.e. it does not show any new interaction between the macroscopic fluctuations. This result yields, for sufficiently short range interactions and for states which are sufficiently clustering, a rigorous proof of the physicist's common belief about the asymptotic simplification that may occur for macroscopic observables.

Finally, having established the macrodynamics $\tilde{\alpha}_{t}$, we apply it to study the case of an equilibrium state, in particular we assume that the microstate $\omega$ is an $\alpha_{t}$-KMS state at inverse temperature $\beta$. We prove that the quasi-free macrostate $\tilde{\omega}$ is then necessarily a $\tilde{\alpha}_{t}$-KMS state at the same temperature. This statement constitutes a proof of one of the basic assumptions of the phenomenological theory of Onsager $[7,8]$ about small oscillations around equilibrium. 


\section{The Model}

We develop the theory for systems which are defined on a $v$-dimensional lattice $\mathbb{Z}^{v}$ and which have a quasi-local structure [9]. Let $\mathscr{D}\left(\mathbb{Z}^{v}\right)$ be the directed set of finite subsets of $\mathbb{Z}^{v}$, where the direction is the inclusion. With each $x \in \mathbb{Z}^{v}$ we associate the algebra $\mathscr{A}_{x}$, a copy of a matrix algebra $M_{N}$ of $N \times N$ matrices. For all $\Lambda \in \mathscr{D}\left(\mathbb{Z}^{v}\right)$, consider the tensor product $\mathscr{A}_{A}=\bigotimes \mathscr{A}_{x}$. The family $A_{\Lambda}, \Lambda \in \mathscr{D}\left(\mathbb{Z}^{v}\right)$ has the usual properties of locality and isotony: $x \in \Lambda$

$$
\begin{array}{ccc}
{\left[\mathscr{A}_{\Lambda_{1}}, \mathscr{A}_{\Lambda_{2}}\right]=0} & \text { if } & \Lambda_{1} \cap \Lambda_{2}=\emptyset, \\
\mathscr{A}_{\Lambda_{1}} \cong \mathscr{A}_{\Lambda_{2}} & \text { if } & \Lambda_{1} \cong \Lambda_{2} .
\end{array}
$$

Denote by $\mathscr{A}_{L}$ all local observables

$$
\mathscr{A}_{L}=\bigcup_{\Lambda \in \mathscr{Q}\left(\mathbb{Z}^{v}\right)} \mathscr{A}_{\Lambda}
$$

The norm closure $\mathscr{B}$ of $\mathscr{A}_{L}$ is again a $C^{*}$-algebra:

$$
\mathscr{B}=\overline{\mathscr{A}}_{L}=\overline{\bigcup_{\Lambda \in \mathscr{P}\left(\mathbb{Z}^{v}\right)} \mathscr{A}_{\Lambda}} \text {, }
$$

and considered as the algebra of quasi-local observables of our system.

The group $\mathbb{Z}^{v}$ of space-translations of the lattice acts as a group of $*$-automorphisms on $\mathscr{B}$ by:

$$
\tau_{x}: A \in \mathscr{A}_{\Lambda} \rightarrow \tau_{x}(A) \in \mathscr{A}_{A+x} ; \quad x \in \mathbb{Z}^{v} .
$$

The dynamics of our system is determined in the usual way by the local Hamiltonians

$$
H_{\Lambda}=\sum_{X \cong \Lambda} \phi(X) ; \quad \Lambda \in \mathscr{D}\left(\mathbb{Z}^{v}\right)
$$

with

$$
\begin{gathered}
\phi(X) \in \mathscr{A}_{X} \quad \text { for all } \quad X \in \mathscr{D}\left(\mathbb{Z}^{v}\right), \\
\tau_{x} \phi(X)=\phi(X+x) ; \quad x \in \mathbb{Z}^{v},
\end{gathered}
$$

and such that, there exists $\lambda>0$ :

$$
\|\phi\|_{\lambda} \equiv \sum_{0 \in X}|X| N^{2|X|} e^{\lambda d(X)}\|\phi(X)\|<\infty,
$$

where $d(X)=\sup _{x, y \in X}|x-y|$, is the diameter of the set $X$ and $|X|$ is the number of elements in $X$.

For $\Lambda \in \mathscr{D}\left(\mathbb{Z}^{v}\right)$, the local dynamics $\alpha_{t}^{\Lambda}$ is given by

$$
\begin{aligned}
\alpha_{t}^{\Lambda}: \mathscr{A}_{\Lambda} & \rightarrow \mathscr{A}_{\Lambda} \\
\alpha_{t}^{\Lambda}(A) & =e^{i t H_{\Lambda}} A e^{-i t H_{\Lambda}}, \quad A \in \mathscr{A}_{\Lambda} .
\end{aligned}
$$

From (2.5) it follows that the global dynamics $\alpha_{t}$ of $\mathscr{B}$ exists as the following norm limit:

$$
\alpha_{t}=\lim _{\Lambda} \alpha_{t}^{\Lambda}
$$


and one has the following estimate [9, Theorem 6.2.11]:

$$
\left\|\alpha_{t}(A)-\alpha_{t}^{\Lambda}(A)\right\| \leqq\|A\|\left|\Lambda_{0}\right|\left(e^{2|t|\|\phi\|_{\lambda}}-1\right) \sum_{x \in \Lambda^{c}} e^{-\lambda|x|_{0}}
$$

where

$$
|x|_{0}=\min _{y \in \Lambda_{0}}|x-y| ; \quad A \in \mathscr{A}_{\Lambda_{0}} .
$$

By (2.4) one also has $\left[\alpha_{t}, \tau_{x}\right]=0$ for $t \in \mathbb{R}, x \in \mathbb{Z}^{v}$. Finally we consider the $C^{*}$-system $\left(\mathscr{B}, \alpha_{t}, \omega\right)$, where $\omega$ is a state of $\mathscr{B}$ which is space and time translation invariant, i.e. $\omega^{\circ} \tau_{x}=\omega$ for all $x \in \mathbb{Z}^{v}$ and $\omega^{\circ} \alpha_{t}=\omega$ for $t \in \mathbb{R}$. Furthermore we assume that the state has the following space-clustering property: let

$$
\alpha_{\omega}(d)=\sup _{\Lambda, \tilde{\Lambda}} \sup _{\substack{A \in \mathscr{A}_{\Lambda} \\ B \in \mathscr{A}_{\tilde{\Lambda}}}}\left\{\frac{1}{\|A\|\|B\|} \mid \omega(A B)-\omega(A) \omega(B) \| d \leqq d(\Lambda, \tilde{\Lambda})\right\},
$$

we suppose that

$$
\sum_{x \in \mathbb{Z}^{v}} \alpha_{\omega}(|x|)<\infty
$$

Remark that this space-factorization or clustering condition is of the same type as in [10], where the asymptotic orbits of non-interacting Fermi particles are studied. They assume that the cluster function $\alpha_{\omega}$ is a bit stronger than of the logarithmic type, we assume the $L^{1}$-type in (2.8). We can derive the results of this paper under the weaker condition, namely: there exists $\delta>0$ such that

$$
\lim _{N \rightarrow \infty} \sqrt{N} \alpha_{\omega}^{N}\left(N^{(1 / 2 v)-\delta}\right)=0
$$

where

$$
\alpha_{\omega}^{N}(d)=\sup _{\substack{\Lambda, \tilde{\Lambda} \\ A \in \mathscr{A}_{A} \\ B \in \mathscr{A}_{\tilde{\Lambda}}}}\left\{\frac{1}{\|A\|\|B\|}|\omega(A B)-\omega(A) \omega(B)| \mid d \leqq d(\Lambda, \tilde{\Lambda}), \max (|\Lambda|,|\tilde{\Lambda}|) \leqq N\right\} .
$$

One readily checks that (2.8) implies (2.9). However for technical convenience we stick to the condition (2.8).

Remark that the function $\alpha_{\omega}$ has the following immediate properties:

$$
2 \geqq \alpha_{\omega} \geqq 0
$$

and monotonically decreasing

$$
\alpha_{\omega}(d) \leqq \alpha_{\omega}\left(d^{\prime}\right) \text { for } \quad d^{\prime} \leqq d .
$$

\section{Systems with Normal Fluctuations}

Denote by $\Lambda_{n}$ the cube centered around the origin with edges of length $2 n+1$. For any $A \in \mathscr{B}$, the local fluctuation $\tilde{A}^{n}$ of $A$ is given by

$$
\tilde{A}^{n}=\frac{1}{\left|\Lambda_{n}\right|^{1 / 2}} \sum_{x \in \Lambda_{n}} \tau_{x}(A-\omega(A))
$$


In this section we characterize a subset $\mathscr{B}_{0}$ of $\mathscr{B}$ such that for all $A \in \mathscr{B}_{0}$ the central limit

$$
\lim _{n \rightarrow \infty} \tilde{A}^{n}=B_{\omega}(A)
$$

exists. The limits (3.2) are called the fluctuations of the observables of $\mathscr{B}_{0}$. The time evolution of fluctuations will be the subject of Sect. 4. Here we restrict ourselves to the study of the central limit and of the mathematical structure of the algebra of fluctuations.

Definition 3.1. Let $\mathscr{B}_{0}$ be any $*$ subspace of $\mathscr{B}$, the system $\left(\mathscr{B}, \mathscr{B}_{0}, \omega\right)$ is said to have normal fluctuations for $\mathscr{B}_{0}$ if

(i) $\forall A, B \in \mathscr{B}_{0}$

$$
\sum_{x \in \mathbb{Z}^{v}}\left|\omega\left(A \tau_{x} B\right)-\omega(A) \omega(B)\right|<\infty
$$

(ii) $\forall A=A^{*} \in \mathscr{B}_{0} ; t \in \mathbb{R}$

$$
\lim _{n \rightarrow \infty} \omega\left(e^{i t \tilde{A}^{n}}\right)=e^{-\left(t^{2} / 2\right) s_{\omega}(A, A)}
$$

where $s_{\omega}(A, B)=\lim _{n \rightarrow \infty} \operatorname{Re} \omega\left(\left(\tilde{A}^{n}\right)^{*} \tilde{B}^{n}\right)$ for all $A, B$ elements of $\mathscr{B}_{0}$. The limit in (ii) is called the central limit.

Remark that, by (i):

$$
s_{\omega}(A, B)=\operatorname{Re} \sum_{x \in \mathbb{Z}^{\nu}}\left(\omega\left(A^{*} \tau_{x} B\right)-\omega\left(A^{*}\right) \omega(B)\right) .
$$

Theorem 3.2. (Central limit theorem). If the state $\omega$ of $\mathscr{B}$ satisfies the $L^{1}$-spacefactorization condition (2.8) then for all $A, B \in \mathscr{A}_{L, \text { sa }}$ (self-adjoint elements of $\mathscr{A}_{L}$ ).

$$
\begin{aligned}
\lim _{n \rightarrow \infty}\left(e^{i \tilde{A}^{n}} e^{i \tilde{B}^{n}}\right) & =\lim _{n \rightarrow \infty} \omega\left(e^{i(\widetilde{A+B})^{n}}\right) e^{-(1 / 2) \omega\left(\left[\tilde{A}^{n}, \tilde{B}^{n}\right]\right)} \\
& =\exp \left\{-\frac{1}{2} s_{\omega}(A+B, A+B)-(i / 2) \sigma_{\omega}(A, B)\right\},
\end{aligned}
$$

where $\sigma_{\omega}(A, B)=\lim 2 \operatorname{Im} \omega\left(\tilde{A}^{n} \tilde{B}^{n}\right)$. In particular the system has normal fluctuations for $\mathscr{B}_{0}=\mathscr{A}_{L}$.

Proof. Basically this theorem is proved already in [3] and [11] under a slightly different formulation of the conditions. This theorem will be proved if we check that the conditions of Theorem 4.1 of [11] follow from condition (2.8).

We have to prove

(i) that for all $A, B \in \mathscr{A}_{L, s a}$ :

$$
\sum_{x \in \mathbb{Z}^{v}}\left|\omega\left(A \tau_{x} B\right)-\omega(A) \omega(B)\right|<\infty
$$

(ii) that for all $A \in \mathscr{A}_{L, s a}$ :

$$
\lim _{n \rightarrow \infty} \frac{1}{\left|\Lambda_{n}\right|} \omega\left(\left(\tilde{A}^{n}\right)^{4}\right)=0 .
$$

Remark that in $[3,11]$ a slightly stronger condition is assumed. However from the 
proof of Theorem 4.1 of [3] it readily follows that the above condition is sufficient. (iii) Condition (2.8) implies condition (2.9). But this is trivial.

It remains to prove (i) and (ii).

(i) Follows from the observation that if $A, B \in \mathscr{A}_{L}$, then $\exists d \in \mathbb{N}$ such that $A, B \in \mathscr{A}_{\Lambda_{d}}$ and

$$
\begin{aligned}
& \sum_{x \in \mathbb{Z}^{v}}\left|\omega\left(A \tau_{x} B\right)-\omega(A) \omega(B)\right| \\
& \quad \leqq \sum_{x \in \Lambda_{2 d}}\left|\omega\left(A \tau_{x} B\right)-\omega(A) \omega(B)\right|+\|A\|\|B\| \sum_{x \in \mathbb{Z}^{v}} \alpha(|x|)<\infty .
\end{aligned}
$$

(ii) Without loss of generality take $\omega(A)=0$ and for simplicity we work out the proof only in the case $A \in \mathscr{A}_{\{0\}}$. Then by translation invariance

$$
\frac{1}{\left|\Lambda_{n}\right|^{3}} \sum_{x, y, k, l \in \Lambda_{n}}\left|\omega\left(A \tau_{y-x} A \tau_{k-x} A \tau_{l-x} A\right)\right| \leqq \frac{1}{\left|\Lambda_{n}\right|^{2}} \sum_{x, y, z \in \Lambda_{2 n}}\left|\omega\left(A \tau_{x} A \tau_{y} A \tau_{z} A\right)\right| .
$$

Take $q(n)=n^{1 / 2-\varepsilon}$, then

$$
\frac{1}{\left|\Lambda_{n}\right|^{2}} \sum_{x, y, z \in \Lambda_{2 n}} \leqq \frac{1}{\left|\Lambda_{n}\right|^{2}}\left(\sum_{x, y, z \in \Lambda_{q(n)}}+3 \sum_{\substack{x, y \in \Lambda_{q(n)} \\ z \in \Lambda_{q(n)}^{q}}}+3 \sum_{\substack{x \in \Lambda_{q(n)} \\ y, z \in \Lambda_{q(n)}^{c}}}+\sum_{x, y, z \in \Lambda_{q(n)}^{c}}\right)
$$

Now we bound each of these terms. Clearly

$$
\begin{aligned}
& \sum_{\substack{x, y, z \in \Lambda_{q(n)} \\
x, y \in \Lambda_{q(n)} \\
z \in \Lambda_{q(n)}^{c_{q(n)}}}}\left|\omega\left(A \tau_{x} A \tau_{y} A \tau_{z} A\right)\right| \leqq\|A\|^{4}\left|\Lambda_{q(n)}\right|^{3} \\
& \left.\tau_{y} A \tau_{z} A\right)\left.\left|\leqq\|A\|^{4}\right| \Lambda_{q(n)}\right|^{2}\left|\Lambda_{2 n}\right| .
\end{aligned}
$$

\section{Furthermore}

$$
\begin{aligned}
\sum_{\substack{x \in \Lambda_{q(n)} \\
y, z \in \Lambda_{q(n)}^{c}}}\left|\omega\left(A \tau_{x} A \tau_{y} A \tau_{z} A\right)\right| \leqq & \sum\left|\omega\left(A \tau_{x} A \tau_{y} A \tau_{z} A\right)-\omega\left(A \tau_{x} A\right) \omega\left(\tau_{y} A \tau_{z} A\right)\right| \\
& +\left|\omega\left(A \tau_{x} A\right)\right|\left|\omega\left(A \tau_{z-y}(A)\right)\right| \\
\leqq & 2 \sum_{\substack{|y|_{q} \leqq|z|_{q} \\
x \in \Lambda_{q(n)}}} \alpha_{\omega}\left(|y|_{q}\right)\|A\|^{4} \\
& +\left|\Lambda_{q}\right|\left|\Lambda_{2 n}\right| \sum_{y \in \mathbb{Z}^{v}} \alpha_{\omega}(|y|)\|A\|^{4}
\end{aligned}
$$

where $|y|_{q}=d\left(y, \Lambda_{q}\right)$. Hence, this term is bounded by

$$
3\left|\Lambda_{q}\right|\left|\Lambda_{2 n}\right| \sum_{y \in \mathbb{Z}^{v}} \alpha_{\omega}(|y|)\|A\|^{4} .
$$

Finally

$$
\sum_{x, y, z \in \Lambda_{q(n)}^{c}}|\cdots| \leqq 6\|A\|^{4} \sum_{\substack{|x| \leq|y| \leq|\leq| \\ x, y, z \in \Lambda_{q(n)}^{c}}} \alpha_{\omega}(|x|) \leqq 6\|A\|^{4}\left|\Lambda_{2 n}\right|^{2} \sum_{x \in \Lambda_{q(n)}^{c}} \alpha_{\omega}(|x|) .
$$


Using these bounds

$$
\begin{aligned}
\frac{\omega\left(\left(\tilde{A}^{n}\right)^{4}\right)}{\left|\Lambda_{n}\right|} \leqq & \frac{\|A\|^{4}}{\left|\Lambda_{n}\right|^{2}}\left(\left|\Lambda_{q(n)}\right|^{3}+3\left|\Lambda_{q(n)}\right|^{2}\left|\Lambda_{2 n}\right|\right. \\
& \left.+9\left|\Lambda_{q(n)}\right|\left|\Lambda_{2 n}\right| \sum_{y \in \mathbb{Z}^{v}} \alpha_{\omega}(|y|)+6\left|\Lambda_{2 n}\right|^{2} \sum_{x \in \Lambda_{q(n)}^{c}} \alpha_{\omega}(|x|)\right) .
\end{aligned}
$$

Using condition (2.8) and the choice of $q(n)$ above, one checks

$$
\lim _{n \rightarrow \infty} \frac{\omega\left(\left(\tilde{A}^{n}\right)^{4}\right)}{\left|\Lambda_{n}\right|}=0 .
$$

Now we are able to introduce the algebra of normal fluctuations of the system $\left(\mathscr{B}, \mathscr{A}_{L}, \omega\right)$. Consider the symplectic space $\left(\mathscr{A}_{L, \mathrm{sa}}, \sigma_{\omega}\right)$ where $\sigma_{\omega}$ is defined in Theorem 3.2. Denote by $W\left(\mathscr{A}_{L, s a}, \sigma_{\omega}\right)$ the CCR-C*-algebra generated by the Weyl operators $\left\{W(A) \mid A \in \mathscr{A}_{L, s a}\right\}$ and satisfying the product rule

$$
W(A) W(B)=W(A+B) \exp -\frac{i}{2} \sigma_{\omega}(A, B) .
$$

The central limit theorem fixes a representation of this CCR-algebra. This is proved in the following theorem which follows from Theorems 3.2 and 3.3 of [3]. Theorem 3.3. For each $A \in \mathscr{A}_{L, s a}$ the limits $\lim \omega\left(e^{i \tilde{A}^{n}}\right)$ define a quasifree state $\omega_{s}$ of the CCR-C*-algebra by

$$
\omega_{s}(W(A))=\exp -\frac{1}{2} s_{\omega}(A, A) .
$$

Moreover if $\gamma$ is $a^{*}$-automorphism of $\mathscr{B}$ leaving $\mathscr{A}_{L}$ invariant, commuting with the space translations and leaving the state $\omega$ invariant, then $\tilde{\gamma}$ given by

$$
\tilde{\gamma}(W(A))=W(\gamma(A))
$$

defines a quasifree*-automorphism of $W\left(\mathscr{A}_{L, s a}, \sigma_{\omega}\right)$.

The quasifree state $\omega_{s}$ of Theorem 3.3 induces a GNS-triplet $\left(\pi_{s}, \mathscr{H}_{s}, \Omega_{s}\right)$ and yields a von Neumann algebra

$$
\mathscr{M}_{s}=\pi_{s}\left(W\left(\mathscr{A}_{L, s a}, \sigma_{\omega}\right)\right)^{\prime \prime} .
$$

This algebra will be called the algebra of normal (macroscopic) fluctuations.

By the above theorems and by the fact that the representation $\pi_{s}$ is regular we are able to identify

$$
\lim _{n \rightarrow \infty} \omega\left(e^{i \tilde{A}^{n}}\right)=\omega_{s}\left(e^{i B_{\omega}(A)}\right),
$$

where $B_{\omega}(A), A \in \mathscr{A}_{L, s a}$ is a Boson field satisfying the Bose commutation relations

$$
\left[B_{\omega}(A), B_{\omega}(B)\right]=i \sigma_{\omega}(A, B) 1 .
$$

Remark also that we can identify the macroscopic fluctuations in $\omega$ with the Bose field $B_{\omega}(\cdot)$, i.e.

$$
\lim _{n \rightarrow \infty} \tilde{A}^{n}=B_{\omega}(A)
$$


The limit $n \rightarrow \infty$ is the central limit.

The rest of this section is devoted to the study of the von Neumann algebra $\mathscr{M}_{s}(4)$. First we describe the property of coarse graining. Mathematically, coarse graining is the property that the map

$$
A \in \mathscr{A}_{L, s a} \rightarrow B_{\omega}(A)
$$

is not injective, i.e. many micro observables yield the same fluctuation. This follows immediately from the central limit theorem. As a matter of example, it is clear that each observable $A \in \mathscr{A}_{L, s a}$ and its space translate $\tau_{x} A \in \mathscr{A}_{L, s a}$ yield the same fluctuation: $B_{\omega}(A)=B_{\omega}\left(\tau_{x} A\right), x \in \mathbb{Z}^{\nu}$.

Let $\left(\pi_{\omega}, \mathscr{H}_{\omega}, \Omega_{\omega}\right)$ be the GNS-triplet induced by the state $\omega$ and consider the sesquilinear form $\langle\cdot, \cdot\rangle_{s}$ on $\mathscr{H}_{\omega}$ with domain $\pi_{\omega}\left(\mathscr{A}_{L}\right) \Omega_{\omega}$ which we simply denote by $\mathscr{A}_{L}$ :

$$
\begin{aligned}
\langle A, B\rangle_{s} & =s_{\omega}(A, B)+\frac{i}{2} \sigma_{\omega}(A, B) \\
& =\sum_{x \in \mathbb{Z}^{\nu}}\left(\omega\left(A^{*} \tau_{x} B\right)-\omega\left(A^{*}\right) \omega(B)\right) .
\end{aligned}
$$

This form is directly related to the structure of the CCR- $C^{*}$-algebra of fluctuations and its quasifree representation (see Theorem 3.2).

This form defines a topology on $\mathscr{A}_{L}$, which is not comparable with the operator topologies on $\mathscr{A}_{L}$ induced by the state $\omega$. In fact this form is not closable in the (weak, strong, ultraweak, ultrastrong) operator topologies. Indeed take $A \in \mathscr{A}_{L}$, by (2.8) the state $\omega$ is $\tau_{x}$-ergodic and

$$
\text { weak- } \lim _{\Lambda} A_{\Lambda}=\text { weak-lim } \frac{1}{|\Lambda|} \sum_{x \in \Lambda} \tau_{x} A=\omega(A) 1 .
$$

On the other hand the space translation invariance of $\omega$ implies:

$$
\left\langle A_{\Lambda}-A, A_{\Lambda}-A\right\rangle_{s}=0 \text { for all } \Lambda \in \mathscr{D}\left(\mathbb{Z}^{v}\right) .
$$

We call $A$ and $B$ in $\mathscr{A}_{L}$ equivalent, denoted by $A \sim B$ if

$$
\langle A-B, A-B\rangle_{\mathrm{s}}=0 .
$$

Clearly this defines an equivalence relation on $\mathscr{A}_{L}$. In particular, remark that $A \sim \tau_{x} A$ for all $x \in \mathbb{Z}^{v}$. The property of coarse graining is mathematically characterized in the following theorem.

Theorem 3.4. Let $A, B \in \mathscr{A}_{L, s a}$, then the following is equivalent:

(i) $A \sim B$,

(ii) $\pi_{s}(W(A))=\pi_{s}(W(B))$.

Proof. Suppose first (ii) is satisfied, then

$$
\left[\pi_{s}(W(A)), \pi_{s}(W(B))\right]=0 \text { and hence } \sigma_{\omega}(A, B)=0 .
$$

Further 


$$
\begin{aligned}
1 & =\omega_{s}\left(W(A) W(B)^{*}\right)=\omega_{s}(W(A) W(-B)) \\
& =\omega_{s}(W(A-B))=\exp -\frac{1}{2} s_{\omega}(A-B, A-B)
\end{aligned}
$$

or $\langle A-B, A-B\rangle_{s}=0$, proving (i). Conversely, suppose $A \sim B$ then

$$
\frac{1}{4}\left|\sigma_{\omega}(A-B, X)\right|^{2} \leqq\langle A-B, A-B\rangle_{s}\langle X, X\rangle_{s}
$$

implies that $\sigma_{\omega}(A-B, X)=0$ for all $X \in \mathscr{A}_{L}$, i.e. $\pi_{s}(W(A-B)) \in \mathscr{M}_{s}^{\prime}$. Also

$$
\begin{aligned}
\left\|\left(\pi_{s}(W(A-B))-1\right) \Omega_{s}\right\|^{2} & =\omega_{s}((W(A-B)-1) *(W(A-B)-1)) \\
& =2-\omega_{s}(W(A-B))-\omega_{s}(W(B-A)) \\
& =0 .
\end{aligned}
$$

As $\Omega_{s}$ is cyclic for $\mathscr{M}_{s}$, it is separating for $\mathscr{M}_{s}^{\prime}$. Hence

or

$$
\pi_{s}(W(A-B))=1
$$

$$
\pi_{s}(W(A))=\pi_{s}(W(B)) .
$$

Denote by $\left[\mathscr{A}_{L}\right]$ the equivalence classes $\mathscr{A}_{L}$ for the equivalence relation $\sim$. The form $\langle\cdot, \cdot\rangle_{s}$ is a scalar product on $\left[\mathscr{A}_{L}\right]$. Denote by $\mathscr{K}_{\omega}$ the Hilbert space obtained as the completion of $\left[\mathscr{A}_{L}\right]$. Clearly $s_{\omega}$ and $\sigma_{\omega}$ extend continuously to $\mathscr{K}_{\omega}$. Denote by $\mathscr{K}_{\omega}^{\mathrm{Re}}$ the real subspace of $\mathscr{K}_{\omega}$ generated by $\left[\mathscr{A}_{L, s a}\right]$. Now one considers the CCR-C*-algebra $W\left(\mathscr{K}_{\omega}^{\mathrm{Re}}, \sigma_{\omega}\right)$ in the same representation $\pi_{s}$ induced by the state $\omega_{s}$.

Corollary 3.5. With the notations of above one has the following equality:

$$
\mathscr{M}_{s}=\pi_{s}\left(W\left(\mathscr{K}_{\omega}^{\mathrm{Re}}, \sigma_{\omega}\right)\right)^{\prime \prime} .
$$

Proof. Clearly by Theorem 3.4 one has

$$
\pi_{s}\left(W\left(\mathscr{A}_{L, s a}, \sigma_{\omega}\right)\right)=\pi_{s}\left(W\left(\left[\mathscr{A}_{L, s a}\right], \sigma_{\omega}\right)\right)
$$

and the corollary follows immediately Proposition 5 from [12].

Remark that we were able to identify a set $\mathscr{K}_{\omega}$ of microscopic not necessarily strictly local observables which is closed for the $\langle\cdot, \cdot\rangle_{s}$-topology and determined by the quasifree state $\omega_{s}$. By the corollary all elements of $\mathscr{K}_{\omega}^{\mathrm{Re}}$ do have macroscopic fluctuations in $\mathscr{M}_{s}$. This fact will turn out to be of importance in the construction of the dynamics.

Finally by Theorem 3.3 there exists a quasifree automorphism $\tilde{\tau}_{x}$ of the CCR-C*-algebra $W\left(\mathscr{A}_{L, s a}, \sigma_{\omega}\right)$ given by

$$
\tilde{\tau}_{x} W(A)=W\left(\tau_{x} A\right) ; \quad x \in \mathbb{Z}^{v} ; \quad A \in \mathscr{A}_{L, s a} .
$$

But as $A \sim \tau_{x} A$, by Theorem 3.4:

$$
\pi_{s}\left(\tilde{\tau}_{x} W(A)\right)=\pi_{s}(W(A))
$$

and by Corollary 3.5, $\tilde{\tau}_{x}$ acts trivially on $\mathscr{M}_{s}$. In particular $\tilde{\tau}_{x} B_{\omega}(A)=B_{\omega}(A)$ for all $A \in \mathscr{K}_{\omega}^{\mathrm{Re}}$.

In the next section we study the announced but less trivial problem, namely the action of the time evolution group on the fluctuation algebra $\mathscr{M}_{s}$. 


\section{Reversible Dynamics of Fluctuations}

Contrary to the space translations, Theorem 3.3 is not directly applicable with $\gamma=\alpha_{t}$, because with this choice, it is not clear and generally not true that $\alpha_{t} \mathscr{A}_{L} \subseteq \mathscr{A}_{L}$. The other conditions,

$$
\left[\tau_{x}, \alpha_{t}\right]=0 \text { and } \omega \circ \alpha_{t}=\omega
$$

such that $\alpha_{t} \tilde{A}^{n}=\left(\widetilde{\alpha_{t} A}\right)^{n}$, are satisfied, and one is tempted to define the dynamics $\tilde{\alpha}_{t}$ of the fluctuations by the formula

$$
\tilde{\alpha}_{t} B_{\omega}(A)=B_{\omega}\left(\alpha_{t} A\right) .
$$

The difficulty with this formula is that it is unclear whether the central limit of the nonlocal observable $\alpha_{t} A$ exists or not, i.e. can one give a meaning to $B_{\omega}\left(\alpha_{t} A\right)$. Furthermore if $B_{\omega}\left(\alpha_{t} A\right)$ exists it remains to prove that $\left(\tilde{\alpha}_{t}\right)_{t}$ defines a weakly continuous group of *-automorphisms on the fluctuation algebra $\mathscr{M}_{s}$.

First we prove the existence of this central limits under the cluster conditions (2.8) and for a dynamics satisfying the condition (2.5) on the potential. We prove this in an indirect way. Using Corollary 3.5, we show that $\alpha_{t} A \in \mathscr{K}_{\omega}$ for all local observables $A$.

Lemma 4.1. If (2.5) and (2.8) are satisfied then

(i) $\sum_{x \in \mathbb{Z}^{v}}\left|\omega\left(\alpha_{t} A \tau_{x} B\right)-\omega(A) \omega(B)\right|<\infty ; A, B \in \mathscr{A}_{L}$,

(ii) If $\alpha_{t}^{n}(A)=e^{i t H_{A_{n}}} A e^{-i t H_{A_{n}}}$ (see (2.6)), $\Lambda_{n}=[-n, n]^{\nu}$, then

$$
\lim _{n \rightarrow \infty}\left\langle\alpha_{t} A-\alpha_{t}^{n} A, \alpha_{t} A-\alpha_{t}^{n} A\right\rangle_{s}=0
$$

uniformly for $t$ in any compact.

Proof. Denote for $n, k \in \mathbb{N}$ :

$$
\Lambda_{k, k+1}^{n}=\Lambda_{2(k+1) n} \backslash \Lambda_{2 k n} .
$$

First we prove that for $k_{0} \geqq 1$, there exists a constant $C(v)$ :

$$
\sum_{k=k_{0}}^{\infty}\left|\Lambda_{k, k+1}^{n}\right|\left\|\alpha_{t} A-\alpha_{t}^{k n} A\right\| \leqq C(v) \sum_{k=k_{0} n}^{\infty} k^{v}\left\|\alpha_{t} A-\alpha_{t}^{k} A\right\|<\infty .
$$

This follows from the straightforward bounds

$$
\left|\Lambda_{k, k+1}^{n}\right| \leqq 2 v(k+1)^{v-1}(2 n)^{v} \leqq 2 v 4^{v}(k n)^{v}
$$

and from (2.7), the convergence of $\alpha_{t}^{k}$ to $\alpha_{t}$, exponentially. Remark also:

$$
\sum_{k=k_{0}+2}^{\infty}\left|\Lambda_{k, k+1}^{n}\right| \alpha_{\omega}((k-1) n) \leqq C(v) \sum_{k=k_{0} n}^{\infty} k^{v-1} \alpha(k)<\infty .
$$

This is clear from

$$
k+1 \leqq 4(k-2) \quad \text { as } \quad k \geqq 3
$$


the monotonicity of the function $\alpha_{\omega}$ :

$$
n \alpha_{\omega}\left(\left(k^{\prime}+1\right) n\right) \leqq \sum_{l=0}^{n-1} \alpha_{\omega}\left(k^{\prime} n+l\right)
$$

by the consecutive bounds:

$$
\begin{aligned}
\sum_{k=k_{0}+2}^{\infty}\left|\Lambda_{k, k+1}^{n}\right| \alpha_{\omega}((k-1) n) & \leqq \sum_{k=k_{0}+2}^{\infty} 2 v 8^{v} n^{v}(k-2)^{v-1} \alpha_{\omega}((k-1) n) \\
& \leqq 2 v 8^{v} \sum_{k=k_{0}}^{\infty} \sum_{l=0}^{n-1}(k n+l)^{v-1} \alpha_{\omega}(k n+l)=2^{v} 8^{v} \sum_{k=k_{0} n}^{\infty} k^{v-1} \alpha_{\omega}(k),
\end{aligned}
$$

and by (2.8) the boundedness.

Now we proceed to the proof of (i) and (ii). Take $A, B \in \mathscr{A}_{\Lambda_{d}}$ and $\omega(A)=\omega(B)=0$, then

$$
\begin{aligned}
\sum_{x \in \mathbb{Z}^{v}}\left|\omega\left(\alpha_{t} A \tau_{x} B\right)\right| & =\sum_{k=0}^{\infty} \sum_{x \in \Lambda_{k, k+1}^{d}}\left|\omega\left(\alpha_{t} A \tau_{x} B\right)\right| \\
& \leqq \sum_{k=0}^{\infty} \sum_{x \in \Lambda_{k, k+1}^{d}}\left|\omega\left(\left(\alpha_{t}-\alpha_{t}^{k d}\right)(A) \tau_{x} B\right)\right|+\left|\omega\left(\alpha_{t}^{k d} A \tau_{x} B\right)\right| \\
& \leqq \\
& \sum_{k=0}^{\infty}\left|\Lambda_{k, k+1}^{d}\right|\|B\|\left\|\alpha_{t} A-\alpha_{t}^{k d} A\right\| \\
& +\left|\Lambda_{6 d}\right|\|A\|\|B\|+\sum_{k=3}^{\infty} \sum_{x \in \Lambda_{k, k+1}^{d}} \alpha_{\omega}((k-1) d)\|A\|\|B\|
\end{aligned}
$$

and (i) follows from (4.1) and (4.2).

Now we show (ii). Remark first that for all finite $n$, the expression is meaningful, because of (i)

$$
\left\langle\alpha_{t} A, \alpha_{t}^{n} A\right\rangle_{s}
$$

is well defined, and

$$
\left\langle\alpha_{t} A, \alpha_{t} A\right\rangle_{s}=\langle A, A\rangle_{s}
$$

which follows from $\left[\alpha_{t}, \tau_{x}\right]=0$ and $\omega^{\circ} \alpha_{t}=\omega$. Now:

$$
\begin{aligned}
& \left\langle\alpha_{t} A-\alpha_{t}^{n} A, \alpha_{t} A-\alpha_{t}^{n} A\right\rangle_{s} \\
& \quad \leqq\left(\sum_{x \in \Lambda_{6 n}}+\sum_{x \in \Lambda_{6 n}^{c}}\right)\left|\omega\left(\left(\alpha_{t} A-\left(\alpha_{t}^{n} A-\omega\left(\alpha_{t}^{n} A\right)\right)\right) \tau_{x}\left(\alpha_{t} A-\alpha_{t}^{n} A\right)\right)\right| .
\end{aligned}
$$

The first sum $\sum_{x \in \Lambda_{6 n}}$ is bounded by

$$
2\left|\Lambda_{6 n}\right|\left\|\alpha_{t} A-\alpha_{t}^{n} A\right\|^{2}
$$

and tends to zero for $n \rightarrow \infty$ by (2.7) uniformly for $t$ in compacts. The second sum $\sum$ is bounded by the sum $S_{n}^{1}+S_{n}^{2}+2 S_{n}^{3}$, where 


$$
\begin{aligned}
& S_{n}^{1}=\sum_{x \in \Lambda_{6 n}^{c}}\left|\omega\left(\alpha_{t}(A) \tau_{x} \alpha_{t} A\right)\right|, \\
& S_{n}^{2}=\sum_{x \in \Lambda_{6 n}^{c}}\left|\omega\left(\left(\alpha_{t}^{n} A-\omega\left(\alpha_{t}^{n} A\right)\right) \tau_{x} \alpha_{t}^{n} A\right)\right|, \\
& S_{n}^{3}=\sum_{x \in \Lambda_{6 n}^{c}}\left|\omega\left(\alpha_{t}(A) \tau_{x} \alpha_{t}^{n} A\right)\right| .
\end{aligned}
$$

Clearly $\lim _{n \rightarrow \infty} S_{n}^{1}=0$ because of the time invariance of $\omega$ and of the cluster condition (2.5). Also using the cluster function $\alpha_{\omega}$ one gets the time independent bound.

$$
S_{n}^{2}=\sum_{k=3}^{\infty} \sum_{x \in \Lambda_{k, k+1}^{n}}|\cdots| \leqq \sum_{k=3}^{\infty}\left|\Lambda_{k, k+1}^{n}\right| \alpha_{\omega}(2 n(k-1))\|A\|^{2}
$$

tending to zero by (4.2). Further

$$
S_{n}^{3}=\left(\sum_{k=3}^{K}+\sum_{k=K+1}^{\infty}\right)|\cdots| .
$$

Now we prove that one can choose the integer $K$ such that the second sum becomes arbitrary small independent of $n$. Then the first finite sum will be shown to become small for $n$ large.

Using (4.1) and (4.2) the second sum yields

$$
\begin{aligned}
\sum_{k=K+1}^{\infty}|\cdots| \leqq & \sum_{k=K+1}^{\infty} \sum_{x \in \Lambda_{k, k+1}^{n}}\left|\omega\left(\left(\alpha_{t} A-\left(\alpha_{t}^{k n} A-\omega\left(\alpha_{t}^{k n} A\right)\right)\right) \tau_{x} \alpha_{t}^{n} A\right)\right| \\
& +\left|\omega\left(\left(\alpha_{t}^{k n} A-\omega\left(\alpha_{t}^{k n} A\right)\right) \tau_{x} \alpha_{t}^{n} A\right)\right| \\
\leqq & \sum_{k=(K+1) n}^{\infty}\left\{2 C(v) k^{v}\left\|\alpha_{t} A-\alpha_{t}^{k} A\right\|\|A\|+C(v) k^{v-1} \alpha_{\omega}(k)\|A\|^{2}\right\} .
\end{aligned}
$$

For all $\varepsilon>0$, one can choose $K$ such that for all $n \geqq 1$ this bound is smaller than $\varepsilon / 2$. The first sum, using again the triangle inequality:

$$
\sum_{k=3}^{K} \sum_{x \in \Lambda_{k, k+1}}|\cdots| \leqq 2\left|\Lambda_{(K+1) n}\right|\|A\|\left\|\alpha_{t} A-\alpha_{t}^{n} A\right\|+S_{n}^{2}
$$

which tends to zero for large $n$.

Remark that by (2.7) and the time independent bounds obtained from (2.8) the convergence is uniform for $t$ in compacts.

As an immediate consequence of the lemma we have that for all local observables $A \in\left[\mathscr{A}_{L}\right], \alpha_{t} A \in \mathscr{K}_{\omega}$ and if $A \in\left[\mathscr{A}_{L, s a}\right]$ then $\alpha_{t} A \in \mathscr{K}_{\omega}^{\mathrm{Re}}$. In view of Corollary 3.5, $W\left(\alpha_{t} A\right)$ is a well defined element of $\mathscr{M}_{s}$ and as

$$
W\left(\alpha_{t} A\right)=\exp i B_{\omega}\left(\alpha_{t} A\right), \quad A \in\left[\mathscr{A}_{L, s a}\right]
$$

the fluctuation $B_{\omega}\left(\alpha_{t} A\right)$ of $\alpha_{t} A$ exists for all $t \in \mathbb{R}$; in other words the time evolved local observables have normal fluctuations if the local observables themselves have.

Before we show the existence of a dynamics on the algebra of fluctuations $\mathscr{M}_{s}$, we define the dynamics on the test function space $\mathscr{K}_{\omega}$. 
Lemma 4.2. The map $U_{t}:\left[\mathscr{A}_{L}\right] \rightarrow \mathscr{K}_{\omega}, U_{t} A=\alpha_{t} A$ is a well defined linear operator on the Hilbert space $\left(\mathscr{K}_{\omega},\langle\cdot, \cdot\rangle_{s}\right)$ extending to a unitary operator for all $t \in \mathbb{R}$. The map $t \rightarrow U_{t}$ is a strongly continuous one-parameter group.

Proof. Remark first that $U_{t}$ is well defined because if $A \sim B$ then by time invariance of the state $\omega$ :

$$
\left\langle\alpha_{t} A-\alpha_{t} B, \alpha_{t} A-\alpha_{t} B\right\rangle_{s}=\langle A-B, A-B\rangle_{s}=0 .
$$

Also by the time invariance for all $A \in\left[\mathscr{A}_{L}\right]$ :

$$
\left\langle U_{t} A, U_{t} A\right\rangle_{s}=\langle A, A\rangle_{s},
$$

yielding that $U_{t}$ is a densely defined isometric operator. The unitarity follows from $U_{t}^{-1}=U_{-t}$. Again using the time invariance and the unitarity of $U_{t}$ for all $t \in \mathbb{R}$, one has for $t, t^{\prime} \in \mathbb{R}$ :

$$
\left\langle U_{t+t^{\prime}} A, B\right\rangle_{s}=\left\langle\alpha_{t+t^{\prime}} A, B\right\rangle_{s}=\left\langle\alpha_{t^{\prime}} A, \alpha_{-t} B\right\rangle_{s}=\left\langle U_{t^{\prime}} A, U_{-t} B\right\rangle_{s}=\left\langle U_{t} U_{t^{\prime}} A, B\right\rangle_{s},
$$

proving the group property: $U_{t} U_{t^{\prime}}=U_{t+t^{\prime}}$.

Finally the continuity of the map follows from the following argument. Take any $A \in \mathscr{A}_{\Lambda_{d}}$ with $\omega(A)=0$ and denote

$$
F_{n}(t)=\left\langle\alpha_{t}^{n} A, A\right\rangle_{s} ; \quad n \geqq d,
$$

then

$$
\begin{aligned}
\left|F_{n}(t)-F_{n}(s)\right| & \leqq\left(\sum_{x \in \Lambda_{n+d}}+\sum_{x \in \Lambda_{n+d}^{c}}\right)\left|\omega\left(\left(\alpha_{t}^{n}-\alpha_{s}^{n}\right)(A) \tau_{x} A\right)\right| \\
& \leqq\left\{\left|\Lambda_{n+d}\right|+\sum_{x \in \mathbb{Z}^{v}} \alpha_{\omega}(|x|)\right\}\|A\|\left\|\alpha_{t}^{n} A-\alpha_{s}^{n} A\right\|,
\end{aligned}
$$

and the continuity of $t \rightarrow F_{n}(t)$ follows from the normcontinuity of the $\alpha_{t}^{n}$. But by Lemma 4.1

$$
\left\langle\alpha_{t} A, A\right\rangle_{s}=\lim _{n}\left\langle\alpha_{t}^{n} A, A\right\rangle_{s}=\lim _{n} F_{n}(t)
$$

uniformly for $t$ in compacts. Hence the continuity of $t \rightarrow\left\langle\alpha_{t} A, A\right\rangle_{s}$ is guaranteed and

$$
\lim _{t \rightarrow 0}\left\langle U_{t} A-A, U_{t} A-A\right\rangle_{s}=0
$$

Now we are able to formulate our main result about the existence of the dynamics of fluctuations.

Theorem 4.3. For all elements $A \in \mathscr{K}_{\omega}^{\mathrm{Re}}$, define $\tilde{\alpha}_{t} W(A)=W\left(U_{t} A\right)$. Then $\tilde{\alpha}_{t}$ extends to a weakly continuous one-parameter group of *-automorphisms of $\mathscr{M}_{s}$.

Proof. From Lemma 4.2 the set $\left(U_{t}\right)_{t \in \mathbb{R}}$ is a group of symplectic maps of $\left(\mathscr{K}_{\omega}^{\mathrm{Re}}, \sigma_{\omega}\right)$. The theorem follows from Corollary 3.5 and Theorem 3.3 adapted to this situation.

It is instructive to realize that this theorem is a non-trivial extension of the time evolution of the fluctuations in case the microdynamics is of the mean field 
type $[2,4,5,6]$. The essential property of mean field theories is that

$$
\alpha_{t} \mathscr{A}_{L} \subseteq \mathscr{A}_{L},
$$

i.e. local observables remain strictly local under the time evolution. Typically for really interacting systems $\alpha_{t} \mathscr{A}_{L} \nsubseteq \mathscr{A}_{L}$ for all finite times $t$. All this means that for mean fields Theorem 3.3 is directly applicable, while for real interacting systems one has to control the time evolution all the way through the central limit. This is done in the preceding lemmas.

The theorem above yields also a rigorous proof of the fact that on the level of fluctuations the time evolution is a linear process. This is expressed essentially by the formula

$$
\tilde{\alpha}_{t} B_{\omega}(A)=B_{\omega}\left(U_{t} A\right) ; \quad A \in \mathscr{K}_{\omega}
$$

with $B_{\omega}$ a representation of a Boson field, which is a linear map. The Bose field $B_{\omega}$ is induced by a quasi-free state, which is a noncommutative version of a gaussian distribution. Hence the gaussian character of the fluctuation observables is expressed by the quasi-free character of the dynamics. Here we studied the reversible dynamics, which is the basis of the linear response theory and the Kubo formula. We come back to this application on another occasion. The above linearity of the dynamics is not only expected for the reversible but also for the irreversible dynamics. This has already been worked out for mean field theories [7], where we derived the Onsager reciprocal relations.

In this paper we considered the time evolution of one type of macroscopic observables, namely the fluctuations. There exists another important type of macroscopic observables, namely the observables at infinity. The algebra of observables at infinity however is commutative and very well known and understood [1]. The typical, and for statistical mechanics may be the most important, example is the algebra $\mathscr{Z}_{\omega}^{\infty}$ of intensive observables of the system consisting of the space means of the local observables; for any $A \in \mathscr{A}_{L}$ one considers

$$
A_{\omega}^{\infty}=\lim _{n \rightarrow \infty} \frac{1}{\left|\Lambda_{n}\right|} \sum_{x \in \Lambda_{n}} \tau_{x} A,
$$

where the limit is taken in the weak operator topology induced on the GNSrepresentation of the state $\omega$ (operator form of the weak law of large numbers). Now one can ask for the time evolution or the dynamics of this algebra $\mathscr{Z}_{\omega}^{\infty}$.

If the state $\omega$ satisfies the cluster condition (2.8) or more generally if the state is ergodic for the space translations, then the algebra $\mathscr{Z}_{\omega}^{\infty}$ of observables at infinity is trivial, i.e. for all $A \in \mathscr{A}_{L}$ or even for $A \in \mathscr{B}$ :

$$
A_{\omega}^{\infty}=\omega(A) \mathbb{1},
$$

and if furthermore the state $\omega$ is time invariant, the time evolution is also trivial: i.e.

$$
\left(\alpha_{t} A\right)_{\omega}^{\infty}=\omega\left(\alpha_{t} A\right) \mathbb{1}=\omega(A) \mathbb{1}=A_{\omega}^{\infty} .
$$

Even if one relaxes the condition (2.5) on the dynamics to allow long range interactions, but such that the $\alpha_{t}$ are still weakly continuous, the dynamics of the observables at infinity remains trivial. 
Tentatives to study the dynamics of the observables at infinity have been done before $[13,14]$. The least that one can say is that it is unclear how one gets a nontrivial dynamics without relaxing the condition of time invariance of the state $\omega$. But the latter is another story. For mean field models this has been worked out in [15].

In this work we proved that the time invariance of the state does not imply the time invariance of the fluctuations. However the time invariance of the microstate: $\omega \circ \alpha_{t}=\omega$ does imply the time invariance of the macro state: $\omega_{s} \circ \tilde{\alpha}_{t}=\omega_{s}$. Now we go one step further. We suppose that $\omega$ is a $\alpha_{t}$-KMS state of $\mathscr{B}$ at inverse temperature $\beta>0$; i.e. for any pair $A, B \in \mathscr{B}$ there exists a complex function $F_{A, B}$ which is analytic on the strip $D_{\beta}=\{z \mid z \in \mathbb{C}, 0<\operatorname{Im} z<\beta\}$, bounded and continuous on the closure $\bar{D}_{\beta}$ and such that

$$
F_{A, B}(t)=\omega\left(A \alpha_{t} B\right), \quad F_{A, B}(t+i \beta)=\omega\left(\alpha_{t}(B) A\right)
$$

for all $t \in \mathbb{R}$.

Using the notations of above, we denote by $\mathscr{K}_{\omega, \alpha}$ the subspace of $\mathscr{K}_{\omega}$ generated by the set

$$
\begin{aligned}
& \left\{A\left(f_{n}^{u}\right) \mid A\left(f_{n}^{u}\right)=\int d t f_{n}^{u}(t) U_{t} A ; n \in \mathbb{N}_{0}, A \in \mathscr{A}_{L}, f_{n}^{u}(t)=f_{n}(t-u) ;\right. \\
& \left.f_{n}(t)=\sqrt{(n / \pi)} e^{-n t^{2}}, u \in \mathbb{R}\right\}(*) .
\end{aligned}
$$

Using the property that $\left\{f_{n}\right\}_{n}$ is a $\delta$-convergent sequence, a standard argument implies that $\mathscr{K}_{\omega, \alpha}$ is dense in $\mathscr{K}_{\omega}$. It is well known [9] that the elements of $\mathscr{K}_{\omega, \alpha}$ are analytic vectors for $\left(U_{t}\right)_{t \in \mathbb{R}}$, i.e. $\forall \psi \in \mathscr{K}_{\omega, \alpha}, \exists F_{\psi}: \mathbb{C} \rightarrow \mathscr{K}_{\omega}$ such that $F_{\psi}(t)=U_{t} \psi$ and for all $\phi \in \mathscr{K}_{\omega}: z \rightarrow\left\langle\phi, F_{\psi}(z)\right\rangle_{s}$ is analytic. Denote $F_{\psi}(z)=U_{z} \psi$. In particular

$$
U_{z} A\left(f_{n}\right)=\int d t f_{n}(t-z) U_{t} A ; \quad A \in \mathscr{A}_{L}
$$

and

$$
U_{t} A\left(f_{n}\right)=A\left(f_{n}^{t}\right) \text {. }
$$

Lemma 4.4. Let $\omega$ be a $\alpha_{t}$-KMS state at $\beta>0$, then for all $A\left(f_{n}\right), B\left(f_{m}\right) \in \mathscr{K}_{\omega}$, the map $z \rightarrow\left\langle A^{*}\left(f_{n}\right), U_{s} B\left(f_{n}\right)\right\rangle_{s}$ is entirely analytic, bounded on $D_{\beta}$ and

$$
\left\langle A^{*}\left(f_{n}\right), U_{t+i \beta} B\left(f_{n}\right)\right\rangle_{s}=\left\langle U_{t} B^{*}\left(f_{n}\right), A\left(f_{n}\right)\right\rangle_{s} .
$$

Proof. For $A, B \in \mathscr{A}_{L}$ with $\omega(A)=\omega(B)=0$,

$$
\left\langle A^{*}\left(f_{n}\right), U_{z} B\left(f_{m}\right)\right\rangle_{s}=\int d t d t^{\prime} f_{n}(t) f_{m}\left(t^{\prime}-z\right)\left\langle U_{t} A^{*}, U_{t^{\prime}} B\right\rangle_{s} .
$$

For $z \in \bar{D}_{\beta}$ one has the bound:

$$
\left|\left\langle A^{*}\left(f_{n}\right), U_{z} B\left(f_{m}\right)\right\rangle_{s}\right| \leqq\left\|f_{n}\right\|_{1}\left\|f_{m}\right\|_{1}\left\|A^{*}\right\|_{s}\|B\|_{s} e^{m \beta^{2}} .
$$

It remains to prove the boundary condition. By Lemma 4.1:

$$
\left\langle A^{*}\left(f_{n}\right), U_{z} B\left(f_{m}\right)\right\rangle_{s}=\int d t d t^{\prime} f_{n}(t) f_{m}\left(t^{\prime}-z\right) \sum_{x \in \mathbb{Z}^{v}} \omega\left(\alpha_{t}(A) \tau_{x} \alpha_{t^{\prime}} B\right) .
$$

Denote

$$
\mathscr{G}_{N}\left(t, t^{\prime}\right)=\sum_{x \in \Lambda_{N}} \omega\left(\alpha_{t}(A) \tau_{x} \alpha_{t^{\prime}} B\right)
$$


then from the proof of Lemma 4.1 (i) one reads off:

$$
\left|\mathscr{G}_{N}\left(t, t^{\prime}\right)\right| \leqq \sum_{x \in \mathbb{Z}^{v}}\left|\omega\left(\alpha_{t} A \tau_{x} \alpha_{t^{\prime}} B\right)\right| \leqq C_{1}+C_{2} e^{M\left(|t|+\left|t^{\prime}\right|\right)}
$$

where $C_{1}, C_{2}$ and $M$ are constants. As this exponential behaviour is dominated by the Gaussians $f_{n}, f_{m}$, one applies the Lebesgue dominated convergence theorem to conclude that

$$
\left\langle A^{*}\left(f_{n}\right), U_{z}\left(f_{m}\right)\right\rangle_{s}=\sum_{x \in \mathbb{Z}^{v}} \omega\left(A\left(f_{n}\right) \tau_{x} \alpha_{z} B\left(f_{m}\right)\right) .
$$

Now using $\left[\tau_{x}, \alpha_{t}\right]=0$, and the $\alpha_{t}$-KMS condition for $\omega$ and $\omega^{\circ} \tau_{x}=\omega$ :

$$
\omega\left(A\left(f_{n}\right) \tau_{x} \alpha_{t+i \beta} B\left(f_{m}\right)\right)=\omega\left(\alpha_{t} B\left(f_{m}\right) \tau_{-x} A\left(f_{n}\right)\right)
$$

Hence

$$
\left\langle A^{*}\left(f_{n}\right), U_{t+i \beta} B\left(f_{m}\right)\right\rangle_{s}=\sum_{x \in \mathbb{Z}^{v}} \omega\left(\alpha_{t} B\left(f_{m}\right) \tau_{x} A\left(f_{n}\right)\right)
$$

and again by the above argument

$$
\left\langle A^{*}\left(f_{n}\right), U_{t+i \beta} B\left(f_{m}\right)\right)_{s}=\left\langle U_{t} B^{*}\left(f_{m}\right), A\left(f_{n}\right)\right\rangle_{s} .
$$

Now we prove our main application of Theorem 4.3. We prove that if the microsystem is in an equilibrium state, then also the macrosystem of fluctuations is in an equilibrium state for the dynamics constructed above, i.e. the notion of equilibrium is preserved under the operation of coarse graining induced by the central limit.

Theorem 4.5. If $\omega$ is a $\alpha_{t}$-KMS state of $\mathscr{B}$ at $\beta>0$, then $\omega_{s}$ is a $\tilde{\alpha}_{t}-\mathrm{KMS}$ state of the von Neumann algebra $\mathscr{M}_{s}$ at the same temperature.

Proof. Denote by $\mathscr{M}_{s, \tilde{\alpha}}$ the subalgebra of $\mathscr{M}_{s}$ generated by the set $\left\{W(\psi) \mid \psi \in \mathscr{K}_{\omega, \alpha}^{\mathrm{Re}}\right\}$. Clearly $\mathscr{M}_{s, \tilde{\alpha}}$ is *-invariant, $\tilde{\alpha}_{t}$-invariant and weakly dense in $\mathscr{M}_{s}$. For $A, B$ observables of the type $(*)$ define the function

$$
\mathscr{G}_{A, B}(t)=\omega_{s}\left(W(A) \tilde{\alpha}_{t} W(B)\right) .
$$

By Theorems 3.2 and 4.3

$$
\mathscr{G}_{A, B}(t)=\exp \left\{-\frac{1}{2}\left\langle A+U_{t} B, A+U_{t} B\right\rangle_{s}+\frac{1}{2} i \sigma_{\omega}\left(A, U_{t} B\right)\right\} .
$$

By Lemma 4.4 this function is analytic on $D_{\beta}$, bounded and continuous on $\bar{D}_{\beta}$ and

$$
\mathscr{G}_{A, B}(t+i \beta)=\omega_{s}\left(\tilde{\alpha}_{t}(W(B)) W(A)\right) .
$$

Using the canonical commutation relations (3.3) one extends trivially these properties to the function

$$
\mathscr{G}_{\psi, \phi}(t)=\omega_{s}\left(W(\psi) \tilde{\alpha}_{t} W(\phi)\right)
$$

for all $\psi, \phi \in \mathscr{K}_{\omega, \alpha}^{\mathrm{Re}}$. By linearity the result extends to the function

$$
t \rightarrow \omega_{s}\left(X \tilde{\alpha}_{t} Y\right) ; \quad X, Y \in \mathscr{M}_{s, \tilde{\alpha}} .
$$

Finally, as $\mathscr{M}_{s, \tilde{\alpha}}$ is dense in $\mathscr{M}_{s}$ and $\omega_{s} \circ \tilde{\alpha}_{t}=\omega_{s}$ for all $t \in \mathbb{R}$ a standard procedure 
([9] proof of Proposition 5.3.7) yields the extension to the function

$$
t \rightarrow \omega_{s}\left(X \tilde{\alpha}_{t} Y\right) \text { for } X, Y \in \mathscr{M}_{s} .
$$

This finishes the proof of the theorem.

\section{References}

1. Lanford, O. E., Ruelle, D.: Commun. Math. Phys. 13, 194 (1969)

2. Goderis, D., Verbeure, A., Vets, P.: Prob. Th. Rel. Fields 82, 527 (1989)

3. Goderis, D., Vets, P.: Commun. Math. Phys. 122, 249 (1989)

4. Hepp, K., Lieb, E. H.: Helv. Phys. Acta 46, 573 (1974)

5. Wreszinski, W. F.: Helv. Phys. Acta 46, 844 (1974)

6. Goderis, D., Verbeure, A., Vets, P.: J. Math. Phys. 29, 2581 (1988)

7. Goderis, D., Verbeure, A., Vets, P.: J. Stat. Phys. 56, 721 (1989)

8. De Groot, S. R., Mazur, P.: Nonequilibrium thermodynamics, Amsterdam: North-Holland 1962

9. Bratteli, O., Robinson, D. W.: Operator algebras and quantum statistical mechanics, vols I, II. Berlin, Heidelberg, New York: Springer 1979/1981

10. Haag, R., Kadison, R. V., Kastler, D.: Commun. Math. Phys. 33, 1 (1973)

11. Goderis, D., Verbeure, A., Vets, P.: Quantum central limit and coarse graining, to appear in: Quantum probability and applications V. Lecture Notes in Mathematics. Berlin, Heidelberg, New York: Springer

12. Manuceau, J., Verbeure, A.: Commun. Math. Phys. 9, 293 (1968)

13. Morchio, G., Strocchi, F.: Commun. Math. Phys. 99, 153 (1985)

14. Morchio, G., Strocchi, F.: Math. Phys. 28, 622 (1987)

15. Bona, P.: J. Math. Phys. 29, 2223 (1988)

Communicated by H. Araki

Received June 21, 1989; in revised form August 18, 1989 
